\title{
A NOTE ON $L$-GROUPS
}

by H. H. TEH

The subject of this note is the study of conditions under which an $l$-group is simply ordered. We start with two definitions: A po-group is called positively related if every pair of elements $a, b>0$ has a lower bound $c>0$. Evidently a po-group is positively related if and only if it is negatively related in the sense that every pair of negative elements $a, b$ has a negative upper bound. A po-group is called a weak l-group if every pair of elements $a, b$ has a maximal lower bound $c$ in the sense that $a, b \geqq c$ and $d>c$ imply that $d$ is not a lower bound of $a, b$. Evidently every weak $l$-group is directed and every $l$-group is a weak $l$-group.

Theorem. A weak l-group $G$ is simply ordered if it is positively related.

Proof. Let $a, b>0$ and $c$ a maximal lower bound of $a, b$. Then $a-c \geqq 0$, $b-c \geqq 0$. We assert that either $a-c=0$ or $b-c=0$. For $a-c, b-c>0$ implies the existence of an element $d>0$ such that $a-c, b-c \geqq d$, i.e. $a, b \geqq d+c>c$, which contradicts the definition of $c$. Therefore we have either $a-c=0$, whence $b \geqq a$ or $b-c=0$, whence $a \geqq b$. Therefore every two positive elements of $G$ are comparable, and, since $G$ is directed, it is simply ordered. This proves the theorem.

Corollary 1. An l-group is simply ordered if it is positively related.

Corollary 2. An l-group is simply ordered if $a, b>0$ implies $a \cap b \neq 0$.

Corollary 3. An l-group is simply ordered if $a \cap b=0$ implies $a=0$ or $b=0$; that is, if every positive element is a weak unit.

Corollary 4. An l-group is simply ordered if $a, b>0$ implies $a+b>a \cup b$.

Proof. This follows from the well-known equality $a \cup b=a-(a \cap b)+b$.

Corollary 5. An l-group is simply ordered if it has a positive element e>0 such that for each $a>0$ there exists some integer $n$ such that na $\geqq e$.

Proof. For each $a, b>0$, let $n a, m b \geqq e$. Evidently $n a \cap m b \geqq e>0$, which implies that $a \cap b>0$, since it is well known that $a \cap b=0$ implies panqb $=0$ for all $p, q=1,2, \ldots$

Corollary 6. Every strongly Archimedean l-group is simply ordered.

(By strongly Archimedean we mean that for every two elements $a, b>0$ there exists some integer $n$ such that $n a \geqq b$.)

To end this note we give an example to show that there exists a positively 
related po-group which is not simply ordered. Let $G=\{(a, b, c) \mid a, b, c$ integers\}, then $G$ forms a group under component-wise addition. Define a partial order in $G$ by writing $(a, b, c) \geqq(0,0,0)$ if and only if either $a>0, b \geqq 0$ or $a \geqq 0, b>0$ or $a=0, b=0, c \geqq 0$. It is then easily verified that $G$ is a positively related po-group which is not simply ordered.

\section{REFERENCE}

(1) G. BIRKHoff, Lattice Theory, Revised edn. (New York, 1960).

Department of Mathematics

THE QUEEN'S UNIVERSITY

BELFAST 